\title{
MINIMAL 4-MANIFOLDS FOR GROUPS OF COHOMOLOGICAL DIMENSION 2
}

\author{
by JONATHAN A. HILLMAN
}

(Received 22nd October 1992)

\begin{abstract}
We show that if $\pi$ is a group with a finite 2-dimensional Eilenberg-Mac Lane complex then the minimum of the Euler characteristics of closed 4-manifolds with fundamental group $\pi$ is $2 \chi(K(\pi, 1))$. If moreover $M$ is such a manifold realizing this minimum then $\pi_{2}(M) \cong \overline{\operatorname{Ext}_{\mathrm{Z}[\pi]}^{2}(Z, Z[\pi])}$. Similarly, if $\pi$ is a $\mathrm{PD}_{3}$-group and $w_{1}(M)$ is the canonical orientation character of $\pi$ then $\chi(M) \geqq 1$ and $\pi_{2}(M)$ is stably isomorphic to the augmentation ideal of $Z[\pi]$.
\end{abstract}

1991 Mathematics subject classification: Primary 57N13, Secondary 57M05.

It is well known that every finitely presentable group is the fundamental group of some closed orientable 4-manifold. Such manifolds are far from unique, for the Euler characteristic may be made arbitrarily large by taking connected sums with simply connected manifolds. Following Hausmann and Weinberger we may define an invariant $q(\pi)$ for any finitely presentable group $\pi$ by $q(\pi)=\min \left\{\chi(M) \mid M\right.$ is a $P D_{4}$-complex with $\left.\pi_{1}(M) \cong \pi\right\}$. We may also define related invariants $q^{X}$ where the minimum is taken over the class of $P D_{4}$-complexes whose normal fibration has an $X$-reduction. An elementary argument shows that $q^{S G}(\pi) \geqq 2-2 \beta_{1}(\pi)+\beta_{2}(\pi)$, where $S G$ is the class of orientable $P D_{4}$-complexes. (See Theorem 1 of $[4]$ ).

In this note we shall show that if $\pi$ has a finite 2-dimensional Eilenberg-MacLane complex than $q(\pi)=2 \chi(K(\pi, 1))$. The class of such groups includes all surface groups and classical link groups, and the groups of many other (bounded) 3-manifolds. The minima are in fact realized by $s$-parallelizable PL (smooth) 4-manifolds. A related argument shows that if $\pi$ is a $P D_{3}$-group and $w_{1}(M)$ agrees with the canonical orientation character of $\pi$ then $\chi(M)>0$ and $\pi_{2}(M)$ is stably isomorphic to the augmentation ideal of $Z[\pi]$. We conclude with some observations on the problem of the uniqueness up to homotopy type or up to homeomorphism of minimal manifolds for groups of cohomological dimension 2. In particular, the algebraic 2-type of a minimal manifold for such a group is determined by the group and the orientation character alone; for surface groups the second Stiefel-Whitney class is the only other invariant needed to determine the full homotopy type of a minimal manifold.

If $R$ is either $Z$ or $Q$ and $H$ is a left $R[\pi]$-module $\bar{H}$ shall denote the conjugate right $R[\pi]$-module with the same underlying abelian group and $R[\pi]$-action given by $h \cdot g=w(g) g^{-1} \cdot h$, for all $h \in H$ and $g \in \pi$, where $w=w_{1}(M)$, considered as a homomorphism from $\pi$ to $\{ \pm 1\}$. (We shall also use the overbar to denote the conjugate of a right 
$R[\pi]$-module). The conjugate of a free left (right) module is a free right (left) module of the same rank. Two $R[\pi]$-modules $H$ and $H^{\prime}$ are stably isomorphic if $H \oplus R[\pi]^{a} \cong H^{\prime} \oplus$ $R[\pi]^{b}$ for some $a, b \geqq 0$.

If the augmentation $Q[\pi]$-module has a finite resolution $P_{*}$, where $P_{i} \cong Q[\pi]^{p_{i}}$, for all $i \geqq 0$ then the alternating sum $\chi(\pi)=\sum_{i \geqq 0}(-1)^{i} p_{i}$ is independent of the resolution. If $K(\pi, 1)$ is homotopy equivalent to a finite complex then $\chi(\pi)=\sum_{i \geqq 0}(-1)^{i} \beta_{i}(\pi ; Q)=$ $\chi(K(\pi, 1))$.

For ease of reading we shall write $e^{i}(H)$ for $\operatorname{Ext}_{Q[\pi]}^{i}(H, Q[\pi])$ or $\operatorname{Ext}_{Z[\pi]}^{i}(H, Z[\pi])$, in Theorems 1 and 2 respectively.

Theorem 1. Let $M$ be a finite $P D_{4}$-complex with fundamental group $\pi$, and suppose that c.d.o $\pi \leqq 2$ and that the augmentation $Q[\pi]$-module has a finite resolution by finitely generated free modules. Then $\chi(M) \geqq 2 \chi(\pi)=2\left(1-\beta_{1}(\pi ; Q)+\beta_{2}(\pi ; Q)\right)$. If moreover $\chi(M)=$

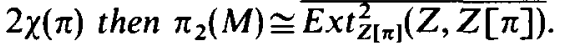

Proof. Since $Q[\pi]$ has global dimension 2, we may assume without loss of generality that there is an exact sequence

$$
0 \rightarrow Q[\pi]^{r} \rightarrow Q[\pi]^{g} \rightarrow Q[\pi] \rightarrow Q \rightarrow 0 .
$$

We may also assume that $M$ is a finite 4-dimensional cell complex. Let $C_{*}$ be the cellular chain complex of the universal covering space $\tilde{M}$, with coefficients $Q$. Then $C_{*}$ is a complex of finitely generated free left $Q[\pi]$-modules, whose homology is $H_{*}=H_{*}(\tilde{M} ; Q)$. Let $H^{t}=H^{t}\left(H o m_{Q[\pi]}\left(C_{*}, Q[\pi]\right)\right)$ be the equivariant cohomology modules. Since $M$ is simply connected and $\pi$ is infinite, $H_{0} \cong Q$ and $H_{1}=H_{4}=0$. The chain complex $C_{*}$ breaks up into exact sequences:

$$
\begin{gathered}
0 \rightarrow C_{4} \rightarrow Z_{3} \rightarrow H_{3} \rightarrow 0, \\
0 \rightarrow Z_{3} \rightarrow C_{3} \rightarrow Z_{2} \rightarrow H_{2} \rightarrow 0, \\
0 \rightarrow Z_{2} \rightarrow C_{2} \rightarrow C_{1} \rightarrow C_{0} \rightarrow Q \rightarrow 0 .
\end{gathered}
$$

The Universal Coefficient spectral sequence (which has $E_{2}^{p q}$ term $\operatorname{Ext}_{Q[\pi]}^{q}\left(H_{p}, Q[\pi]\right)$ and which converges to $H^{p+q}$ ) gives another exact sequence:

$$
0 \rightarrow e^{2} Q \rightarrow H^{2} \rightarrow e^{0} H_{2} \rightarrow 0
$$

and isomorphisms $H^{1} \cong e^{1} Q$ and $e^{1} H_{2}=e^{2} H_{3}=0$. Poincaré duality gives further isomorphisms $H^{1} \cong \bar{H}_{3}, H^{2} \cong \bar{H}_{2}, H^{3}=0$ and $H^{4} \cong \bar{Q}$.

Applying Schanuel's Lemma to the sequences (1) and (4) we obtain $Z_{2} \oplus C_{1} \oplus$ $Q[\pi] \oplus Q[\pi]^{r} \cong C_{2} \oplus C_{0} \oplus Q[\pi]^{g}$, so $Z_{2}$ is a finitely generated stably free module. Similarly, $Z_{3}$ is projective, since p.d. $H_{2} \leqq 2=$ gl.dim. $Q[\pi]$. Since $\pi$ is finitely presentable it is accessible, and hence $e^{1} Q$ is finitely generated as a $Q[\pi]$-module, by Theorems IV.7.5 
and VI.6.3 of [1]. Therefore $Z_{3}$ is also finitely generated, since it is an extension of $H_{3} \cong \overline{e^{1} Q}$ by $C_{4}$. Dualizing the sequence (3) and using the fact that $e^{1} H_{2}=0$ we obtain an exact sequence of right modules

$$
0 \rightarrow e^{0} H_{2} \rightarrow e^{0} Z_{2} \rightarrow e^{0} C_{3} \rightarrow e^{0} Z_{3} \rightarrow e^{2} H_{2} \rightarrow 0 .
$$

Since duals of finitely generated projective modules are projective, it follows that $e^{0} \mathrm{H}_{2}$ is projective. Hence the sequence (5) gives $H^{2} \cong e^{0} H_{2} \oplus e^{2} Q$.

Let $l$ be the augmentation ideal of $Q[\pi]$. Then there are exact sequences

$$
\begin{gathered}
0 \rightarrow Q[\pi]^{r} \rightarrow Q[\pi]^{g} \rightarrow I \rightarrow 0 \text { and } \\
0 \rightarrow I \rightarrow Q[\pi] \rightarrow Q \rightarrow 0 .
\end{gathered}
$$

Dualizing, we obtain exact sequences of right modules

$$
\begin{aligned}
0 \rightarrow e^{0} I & \rightarrow Q[\pi]^{g} \rightarrow Q[\pi]^{r} \rightarrow e^{2} Q \rightarrow 0 \text { and } \\
0 & \rightarrow Q[\pi] \rightarrow e^{0} I \rightarrow e^{1} Q \rightarrow 0 .
\end{aligned}
$$

Applying Schanuel's Lemma twice more, to the pairs of sequences (2) and the conjugate of (10) (using $H_{3} \cong \overline{e^{1} Q}$ ) and to (3) and the conjugate of (9) (using $H_{2} \cong \overline{e^{0} H_{2}} \oplus \overline{e^{2} Q}$ ) and putting all together, we obtain an isomorphism $Z_{3} \oplus\left(Q[\pi]^{2 g} \oplus C_{0} \oplus C_{2} \oplus C_{4}\right) \cong Z_{3} \oplus$ $\left(Q[\pi]^{2+2 r} \oplus C_{1} \oplus C_{3} \oplus \overline{e^{0} H_{2}}\right)$. Since all the summands are finitely generated projective modules, it follows that $\overline{e^{0} H_{2}}$ is stably free. By a theorem of Kaplansky it has a well defined, nonnegative rank (see page 122 of [9]). On adding a projective complement for $Z_{3}$ to each side of this equation and comparing ranks we find that $\overline{e^{0} \mathrm{H}_{2}}$ has rank $\chi(M)-2(1-g+r)$. Thus $\chi(M) \geqq 2 \chi(\pi)$.

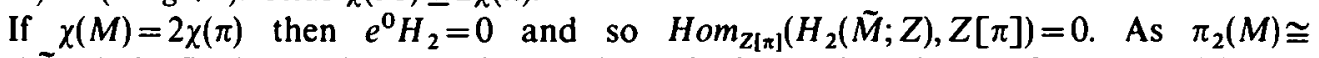
$H_{2}(\tilde{M} ; Z)$ the final assertion now follows from the integral analogue of sequence (5).

This theorem extends Theorem .VII.4 of [5], which assumes also that $\pi$ has one end and a finite 2-dimensional Eilenberg-MacLane space, and that $\chi(\pi)=0$.

Corollary 1. If $\mathrm{H}_{2}(\pi ; Q) \neq 0$ the Hurewicz homomorphism from $\pi_{2}(M)$ to $\mathrm{H}_{2}(M ; Q)$ is nonzero.

Proof. By the theorem, $\mathrm{H}_{2}(M ; Q)$ has dimension at least $2 \beta_{2}(\pi ; Q)$ and so cannot be isomorphic to $H_{2}(\pi ; Q)$ unless both are 0 .

If $H_{2}(\pi ; Q) \neq 0$ the theorem gives a better estimate for $q(\pi)$ than the general estimate mentioned above.

Corollary 2. If $\pi=\pi_{1}(P)$ where $P$ is an aspherical finite 2-complex then $q(\pi)=2 \chi(P)$, 
and the minimum is realized by an s-parallelizable PL 4-manifold. If moreover $\pi$ has one end and $M$ is any closed 4-manifold with $\pi_{1}(M) \cong \pi$ then $\pi_{2}(M)$ is stably isomorphic to $\overline{\operatorname{Ext}_{Z[\pi]}^{1}(Z, Z[\pi])}$. Hence the first $k$-invariant of $M$ is 0 .

Proof. If we choose a PL embedding $j: P \rightarrow R^{5}$, the boundary of a regular neighbourhood $N$ of $j(P)$ is an $s$-parallelizable PL 4-manifold with fundamental group $\pi$ and with Euler characteristic $2 \chi(P)$. By the theorem, this is best possible.

The second assertion follows on reworking the argument of the theorem with $Z[\pi]$ coefficients. As $c . d . \pi \leqq 2$ we have $H^{3}\left(\pi ; \pi_{2}(M)\right)=0$ and so the first $k$-invariant is 0 .

Note that the conjugation of the module structure involves the orientation character of $M$. Is the assumption that $\pi$ has one end needed for the second assertion? By Theorem II.2 of [5] a finitely presentable group is the fundamental group of an aspherical finite 2-complex if and only if it has cohomological dimension $\leqq 2$ and is efficient, i.e. has a presentation of deficiency $\beta_{1}(\pi ; Q)-\beta_{2}(\pi ; Q)$. It is not known whether every finitely presentable group of cohomological dimension 2 is efficient.

Corollary 3. If $\pi$ is the group of an unsplittable $\mu$-component 1-link then $q(\pi)=0$.

For such link groups the general estimate gives only $q^{S G}(\pi) \geqq 1-\mu$. If $\pi$ is the group of a $\mu$-component $n$-link with $n \geqq 2$ then $H_{2}(\pi ; Q)=0$ and so $q(\pi) \geqq 2(1-\mu)$, with equality if and only if $\pi$ is the group of a 2-link. (See Theorem II.5 of [5]).

Corollary 4. If $\pi$ is an extension of $Z$ by a finitely generated free normal subgroup then $q(\pi)=0$. If $M$ is a closed 4-manifold with $\pi_{1}(M) \cong \pi$ then $\chi(M)=q(\pi)$ if and only if $M$ is homotopy equivalent to a manifold which fibres over $S^{1}$ with fibre a closed 3-manifold with free fundamental group.

Proof. Since $\pi$ is the fundamental group of the mapping torus of a self homotopy equivalence of a finite graph, the first assertion follows from Corollary 2, while the second assertion is Theorem 1 of [6].

The homotopy type of such a manifold $M$ is determined by $\pi$ and $w_{1}(M)$. Finitely presentable extensions of $Z$ by free groups of infinite rank can have nonzero Euler characteristic.

Corollary 5. If $\pi$ is the group of a closed aspherical surface $F$ then $q(\pi)=2 \chi(F)$. If $M$ is a closed 4-manifold with $\pi_{1}(M) \cong \pi$ then $\chi(M)=q(\pi)$ if and only if $M$ is homotopy equivalent to the total space of an $S^{2}$-bundle over $F$.

Proof. The final assertion is Theorem 3 of [7].

The homotopy types of such minimal 4-manifolds for each surface group may be distinguished by their Stiefel-Whitney classes [11]. Note that if $F$ is orientable then 
$S^{2} \times F$ is a minimal 4-manifold for $\pi$ which is both $s$-parallelizable and also a projective algebraic complex surface.

Similar arguments give the following theorem.

Theorem 2. Let $M$ be a finite $P D_{4}$-complex whose fundamental group $\pi$ is a $P D_{3}$-group such that $w_{1}(\pi)=w_{1}(M)$. Then $\chi(M)>0$ and $\pi_{2}(M)$ is stably isomorphic to the augmentation ideal $I$ of $Z[\pi]$.

Proof. The cellular chain complex for the universal covering space $\tilde{M}$ gives exact sequences

$$
\begin{aligned}
& 0 \rightarrow C_{4} \rightarrow C_{3} \rightarrow Z_{2} \rightarrow H_{2} \rightarrow 0 \text { and } \\
& 0 \rightarrow Z_{2} \rightarrow C_{2} \rightarrow C_{1} \rightarrow C_{0} \rightarrow Z \rightarrow 0 .
\end{aligned}
$$

Since $\pi$ is a $P D_{3}$-group the augmentation module $Z$ has a finite projective resolution of length 3. On comparing sequence (2) with such a resolution and applying Schanuel's lemma we find that $Z_{2}$ is a finitely generated projective $Z[\pi]$-module. Since $\pi$ has one end, the Universal Coefficient spectral sequence reduces to an exact sequence

$$
0 \rightarrow H^{2} \rightarrow e^{0} H_{2} \rightarrow e^{3} Z \rightarrow H^{3} \rightarrow e^{1} H_{2} \rightarrow 0
$$

and isomorphisms $H^{4} \cong e^{2} H_{2}$ and $e^{3} H_{2}=e^{4} H_{2}=0$. Poincare duality implies that $H^{3}=0$ and $H^{4} \cong \bar{Z}$. Hence (3) reduces to

$$
0 \rightarrow H^{2} \rightarrow e^{0} H_{2} \rightarrow e^{3} Z \rightarrow 0
$$

and $e^{1} H_{2}=0$. Hence on dualizing the sequence (1) we get an exact sequence of right modules

$$
0 \rightarrow e^{0} \mathrm{H}_{2} \rightarrow e^{0} \mathrm{Z}_{2} \rightarrow e^{0} \mathrm{C}_{3} \rightarrow e^{0} \mathrm{C}_{4} \rightarrow e^{2} \mathrm{H}_{2} \rightarrow 0 .
$$

Schanuel's lemma again implies that $e^{0} \mathrm{H}_{2}$ is a finitely generated projective module. By Poincaré duality we may splice together (1) and the conjugate of (4) to get

$$
0 \rightarrow C_{4} \rightarrow C_{3} \rightarrow Z_{2} \rightarrow \overline{e^{0} H_{2}} \rightarrow Z \rightarrow 0 .
$$

(Note that we have used the hypothesis on $w_{1}(M)$ here). Applying Schanuel's lemma once more to the pair of sequences (2) and (6) we obtain $C_{0} \oplus C_{2} \oplus C_{4} \oplus Z_{2} \cong \overline{e^{0} H_{2}} \oplus$ $C_{1} \oplus C_{3} \oplus Z_{2}$. Hence $\overline{e^{0} H_{2}}$ is stably free, of rank $\chi(M)$. Since $\frac{e^{0} H_{2}}{2}$ maps onto $Z$, by (6), $\chi(M)>0$. Since $\pi$ is a $P D_{3}$-group, $e^{3} Z \cong \bar{Z}$ and so the final assertion follows from (4).

Corollary 1. $q(\pi) \leqq 2$. 
Proof. If $M$ is a finite $P D_{4}$-complex with $\pi_{1}(M) \cong \pi$ then the covering space associated with the kernel of $w_{1}(M)-w_{1}(\pi)$ satisfies the condition on $w_{1}$. Since the condition $\chi(M)>0$ is invariant under passage to finite covers, $q(\pi) \geqq 1$.

Let $N$ be a $P D_{3}$-complex with fundamental group $\pi$. We may suppose that $N=N_{0} \cup D^{3}$, where $N_{0} \cap D^{3}=S^{2}$. Let $M=N_{0} \times S^{1} \cup S^{2} \times D^{2}$. Then $M$ is a finite $P D_{4}$-complex, $\chi(M)=2$ and $\pi_{1}(M) \cong \pi$. Hence $q(\pi) \leqq 2$.

If $N$ is an aspherical, closed orientable 3-manifold the manifold $M$ constructed in this corollary is the result of surgery on a loop in $N \times S^{1}$ and is a $S P I N_{4}$-manifold. Since a $S P I N_{4}$-manifold must have even Euler characteristic we see that $q^{S P I N}(\pi)=2$.

Can Theorem 2 be extended to all torsion free 3-manifold groups, or more generally to all free products of $P D_{3}$-groups?

The above results suggest that the algebraic 2-type of a closed 4-manifold whose fundamental group $\pi$ has cohomological dimension 2 and one end may be determined by $\pi, w_{1}(M)$ and $\chi(M)$ alone, for these invariants determine $\pi_{2}(M)$ stably and the $k$-invariant must be 0 in all such cases. For surface groups $w_{2}(M)$ is the only other invariant needed to determine the full homotopy type of a manifold with group $\pi$ and $\chi(M)=q(\pi)=2 \chi(\pi)[7]$. In general we may expect to need stronger information on how the top cell is attached. The signature and the equivariant intersection pairing on $\pi_{2}(M)$ are the obvious candidates.

If we bypass this question and consider classification up to homeomorphism instead we face the problem that the disk embedding theorem is at present only known over elementary amenable groups [2]. If $\pi$ is elementary amenable and c.d. $Q^{\pi} \leqq 2$ then it is locally-finite by solvable, of Hirsch length at most 2, by Lemma 2 and Theorem 2 of [8]. If moreover $\pi$ is torsion free then it is an HNN extension $Z *_{m}$ with a presentation of the form $\left\langle x, y \mid y x y^{-1}=x^{m}\right\rangle$, for some integer $m \neq 0$, or is $Z$, by the Corollary to Theorem 6 of [8]. It can be shown that (if $m$ is even) any homotopy equivalence between two minimal orientable 4-manifolds with such groups is homotopic to a homeomorphism. (Compare Theorem VIII.6 of [5]).

For other fundamental groups we may seek a classification up to $s$-cobordism or stable homeomorphism. Minimal manifolds for surface groups are determined up to $s$ cobordism by their homotopy type. A similar result should hold also when $\pi$ is the group of a finite graph of groups, all of whose vertex groups are infinite cyclic. (Compare the last section of [7]). This class of groups includes free groups, surface groups, the groups $Z *_{m}$ and extensions of $Z$ by finitely generated free normal subgroups.

Two closed 4-manifolds $M$ and $M^{\prime}$ are said to be stably homeomorphic if they become homeomorphic after taking connected sums with copies of $S^{2} \times S^{2}$. The stable homeomorphism classification for orientable 4-manifolds with a given "normal 1-type" $\xi$ may be identified with the quotient $\Omega_{4}(\xi) / A u t(\xi)$ [10]. (See [3] for a brief outline).

Theorem 3. If c.d. $\pi=2$ and $H_{2}(\pi ; Z / 2 Z)=0$ then the stable homeomorphism class of a $\mathrm{SPIN}_{4}$-manifold with fundamental group $\pi$ is determined by its signature.

Proof. The stable homeomorphism classes of $S P I N_{4}$-manifolds with group $\pi$ correspond to the elements of $\Omega_{4}^{S P I N}(K(\pi, 1))[10]$. The hypotheses on $\pi$ imply that the 
projection onto $\Omega_{4}^{S P I N}$ is an isomorphism. The theorem follows, as the signature gives an isomorphism from this group to $Z$.

Corollary. Any two minimal orientable 4-manifolds with fundamental group $\pi$ are stably homeomorphic.

Proof. If $M$ is a closed orientable 4-manifold with $\pi_{1}(M) \cong \pi$ and $\chi(M)=2 \chi(\pi)$ then $H_{2}(M ; Z / 2 Z)=0$ and so $M$ is a $S P I N_{4}$-manifold with signature 0 .

What other invariants are needed if $\tilde{\Omega}_{4}^{S P I N}(K(\pi, 1))=H_{2}(\pi ; Z / 2 Z) \neq 0$ ? Is every $S P I N_{4}$-manifold with signature 0 and with such a fundamental group the stabilization of a minimal manifold? After stabilizing we may assume that $e^{0} \mathrm{H}_{2}$ is a free direct summand of $\pi_{2}(M)$. Can we represent a half basis by disjointly embedded spheres?

Added in proof. Let $\pi$ be the fundamental group of a closed orientable 3-manifold, and suppose that $\pi \cong F * G$ where $F$ is free of rank $r$ and $G$ has no infinite cyclic free factors. If $M$ is an orientable $P D_{4}$-complex with $\pi_{1}(M) \cong \pi$ then the image of $H^{2}(\pi ; Q)=$ $H^{2}(G ; Q)$ in $H^{2}(M ; Q)$ is self-annihilating under cup-product, since c.d. ${ }_{Q} \pi \leqq 3$. Hence $\beta_{2}(M) \geqq 2 \operatorname{dim}_{Q} H^{2}(G ; Q)=2\left(\beta_{1}(M)-r\right)$. It follows easily that $q^{s G}(\pi)=2(1-r)$, and the minimum is realized by a smooth s-parallelizable 4-manifold.

\section{REFERENCES}

1. W. Dicks and M. J. Dunwoody, Groups acting on Graphs (Cambridge studies in advanced mathematics 17, Cambridge University Press, Cambridge-New York-New RochelleMelbourne-Sydney, 1989).

2. M. H. Freedman and F. Quinn, Topology of 4-Manifolds (Princeton University Press, Princeton, New Jersey, 1990).

3. I. Hambleton and M. KRECK, Smooth structures on algebraic surfaces with finite fundamental group, Invent. Math. 102 (1990), 109-114.

4. J.-C. Hausmann and S. Weinberger, Caractérisques d'Euler et groupes fondamentaux des variétés de dimension 4, Comment. Math. Helv. 60 (1985), 139-144.

5. J. A. Hillman, 2-Knots and their Groups (Australian Mathematical Society Lecture Series 5, Cambridge University Press, Cambridge-New York-New Rochelle-Melbourne-Sydney, 1989).

6. J. A. Hillman, A homotopy fibration theorem in dimension four, Topology Appl. 33 (1989), 151-161.

7. J. A. Hillman, On 4-manifolds homotopy equivalent to surface bundles over surfaces, Topology Appl. 40 (1991), 275-286.

8. J. A. Hillman, Elementary amenable groups and 4-manifolds with Euler characteristic $0, J$. Austral. Math. Soc. 50 (1991), 160-170.

9. I. R. Kaplansky, Fields and Rings (Chicago University Press, Chicago-London, 1969).

10. M. KRECK, Surgery and Duality, preprint, Johannes-Gutenberg-Universität Mainz (1985).

11. P. Melvin, 2-sphere bundles over compact surfaces, Proc. Amer. Math. Soc. 92 (1984), 567-572.

School of Mathematics and Statistics

UNIVERSITY OF SYDNEY

SyDNEY, NSW 2006

Australia 